\title{
Vision Neurorehabilitation 2025: A Commitment Towards Advancing Care, Sharing Knowledge and Rebuilding Lives
}

\author{
Isha Suresh Akulwar \\ Department of Neurophysiotherapy, K. J. Somaiya College of Physiotherapy, Mumbai, India \\ Email address: \\ drishasa@yahoo.co.in \\ To cite this article: \\ Isha Suresh Akulwar. Vision Neurorehabilitation 2025: A Commitment Towards Advancing Care, Sharing Knowledge and Rebuilding Lives. \\ International Journal of Neurologic Physical Therapy. Special Issue: Vision Neurorehabilitation 2025: "A Commitment Towards Advancing \\ Care, Sharing Knowledge and Rebuilding Lives”. Vol. 5, No. 2, 2019, pp. 51-62. doi: 10.11648/j.ijnpt.20190502.14
}

Received: August 31, 2019; Accepted: October 22, 2019; Published: December 4, 2019

\begin{abstract}
International evidence shows that people with disabilities have unmet health and rehabilitation needs, face barriers in accessing mainstream health-care services, and consequently have poor quality of life. This article provides a narrative overview of literature on neurorehabilitation in developing countries, especially from the perspective of India and highlights some of the challenges in rehabilitation within the context of neurological conditions. Several issues related to neurorehabilitation are discussed for a systematic approach to build horizontal health care systems that are sustainable and responsive; involvement of bilateral agencies using multi-sectorial approach, new partnerships, strategic collaboration; provision of technical assistance, research and development; and capacity-building for a strong interdisciplinary workforce. Other factors that need to be addressed include governance for strengthening health care systems through logistics, surveillance and service delivery; and adequate scaling. Enlisted here is also a list of comprehensive summary actions to tackle neurological disorder-related disability, and offers governments, policy-makers and other relevant stakeholders a blueprint for implementing recommendations of the World Disability Report and the Convention on the Rights of Persons with Disabilities, and facilitate social inclusion of persons with neurological conditions, in countries at all stages of development. This document is prepared for crystallizing the vision of neurorehabilitation for the future in the year 2025. The vision will reflect our aspirations, the full potentials of growth and development, and layout the efforts needed to fulfill this vision. This vision may in parallel serve to address the strategic implementation for other disability conditions. We hope it will facilitate increased cooperation and innovation and inspire commitment to preventing these debilitating disorders and providing the best possible care for people who suffer from them.
\end{abstract}

Keywords: Disability, Rehabilitation, Neurological Disorder, Developing Country, World Health Organization

\section{Introduction}

Neurological disorders are one of the greatest threats to world public health and a challenge to health care.

Neurological disorders have an effect on one billion people worldwide and constitute $6.3 \%$ of the global burden of disease [1]. The prevalence of disability due to neurological conditions is escalating worldwide with an estimated 92 million disability-adjusted life-years (DALYs) in 2005, which is projected to increase by $12 \%$ to 103 million in 2030 . Despite improved medical care and survivorship, people with long term neurological conditions have significant medium to longer-term functional and psychosocial issues contributing to their complex disabilities that need comprehensive management, including rehabilitation. These add to the economic burden for healthcare systems (particularly in low resourced countries), wherever despite improved acute health care systems, health policies haven't extended to include rehabilitation. Neurorehabilitation is the delivery of a coordinated interdisciplinary care program comprising 'a set of measures that assist individuals who experience (or are likely to experience) disability to achieve and maintain optimal function in interaction with their environment [1], for maximum independence and social reintegration $[2,3]$. The treating team includes neurologists, rehabilitation physicians, nurses and allied health professionals. These programs are individualized and goal-oriented to meet the specific needs of the People with Disability (PwD) and encompass the 
International Classification of Functioning, Disability and Health (ICF) framework, where activity limitation (e.g. mobility, continence, self-care) and participation restriction (e.g. work, driving, community activities) interact with contextual factors (environmental and personal) that influence performance and participatory outcomes [1, 4-6]. Neurorehabilitation is interdisciplinary and cross- sectorial, requiring collaboration in the coordinated efforts of numerous diverse sectors, professions, patients and community. An emerging intellectual paradigm for neurological recovery has brought forward promising Neurorehabilitation approaches. An emerging intellectual paradigm for neurological recovery has brought forward promising Neurorehabilitation approaches. However, Neurorehabilitation is in infancy in India. Looking ahead to the challenges for best of the health and wellbeing of the people we serve - delivering our vision and strategic priorities will require us to change the way we work within our clinical services, and will require us to reshape our resources.

Our 'Neurorehabilitation Vision 2025' is

'To optimize function and quality of life of people with Neurorehabilitation needs through the integration of Education, Quality care, Technology, Research and Advocacy'

Core Values

In India, having an upscale humanistic tradition, we are committed to skilled collaboration, community involvement, integrity, answerability and respect.

Key goals

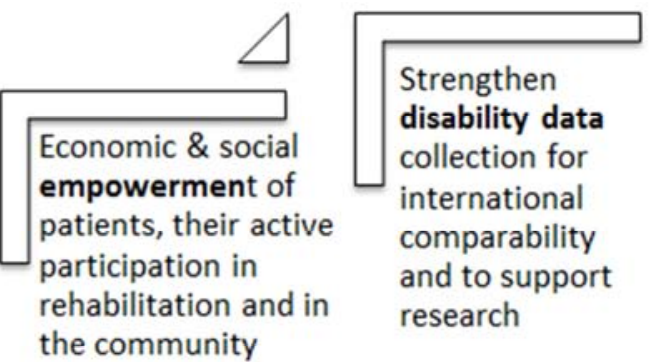

Figure 1. Key Goals of Neurorehabilitation.

\section{Strategic Priorities}

\subsection{Health Care Access for All}

The majority of PwD is economically deprived and experience difficulties in accessing basic health services including rehabilitation [7]. Only 3\% of individuals who need rehabilitation globally receive the service [8], and this is significantly higher for PwD in low-income compared with high-income countries [1]. Majority of our Indian population is rural based where accessibility and affordability are the main barriers.

\subsubsection{Access to Services and Assistive Technologies}

Disability and neurorehabilitation services are limited or often do not exist in rural areas, not only in developing countries but also in some parts of the developed world [1]. As most available services are usually based in urban areas, access to such facilities is often costly, time-consuming and difficult due to lack of transportation [9]. Assistive devices such as canes, crutches, prostheses, wheelchairs etc., are integral part of health care for persons with neurological conditions and widely prescribed to maintain, or improve functional capabilities and participation. In many highincome countries these are provided by national health care systems, through rehabilitation services, insurance companies, charities and NGOs. However, in many low income countries there is still large gap in service provision of these essential disability services $[1,10]$.

\subsubsection{Poverty and Cost of Services}

The cost of access to neurorehabilitation services is a barrier for people with neurological disorders particularly in low income countries, where most services are available only as an out-of pocket payment [11]. Disability can be both a cause and consequence of poverty, which impedes people with disability attaining required services [12]. Many people with neurological disorders (and family) in low-income countries are either unemployed or have lower incomes, compromising adequate care. The persons with neurological disabilities usually depend on family members for help with daily activities, which perpetuates the cycle of poverty [13].

Thus, our first key vision is Health care for all: Our strategy is to bridge a gap in provision of the essential disability services; and to improve the access to rehabilitation services and assistive technologies. [Figure 2] 


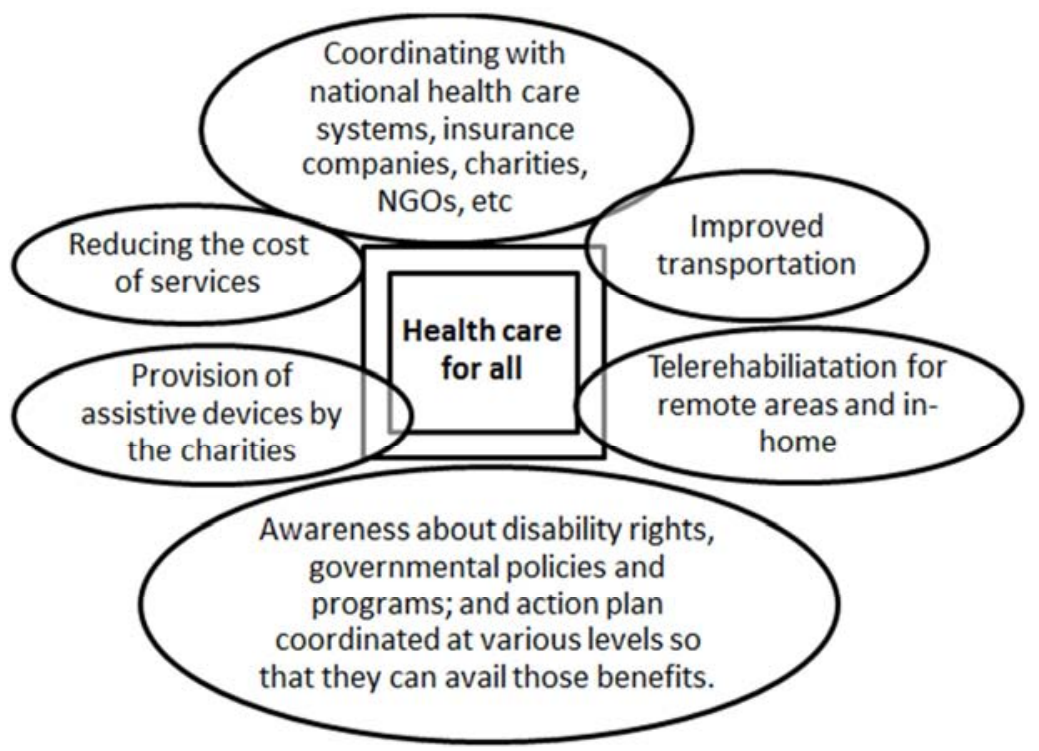

Figure 2. Health care for all.

\subsection{Key Global Initiatives}

The 'Global Burden of Disease' study highlighted neurological disorders as one of the greatest threats to world public health [14]. In response, a number of global public health projects are launched by the WHO, including Global Initiatives on Neurology and Public Health. The WHO in collaboration with the World Federation of Neurology (WFN) in 2004 published the first comprehensive report on country resources for neurological disorders after conducting an international Survey of Country Resources for Neurological Disorders (109 countries, covering over $90 \%$ of world population) [15]. This report highlighted the inadequacy in resources and inequalities in access to care for patients with neurological disorders, especially in those living in the developing world [16]. In 2006, the WHO published: 'Neurological disorders: public health challenges', to inform and help governments, public health institutions,
NGOs and others to formulate public health policies directed at specific neurological disorders; and technical guidelines and policy papers for improved access for PwD (e.g., community-based rehabilitation; manual wheelchairs, training packages etc.) [14].

In April 2014, the WHO global 'Disability Action Plan' (DAP) 2014-2021, provided a comprehensive list of specific actions to achieve the objectives: human- rights based approach (empowerment of PwD); life-course approach (continuum of care); universal health coverage; culturallyappropriate person-centered approach; multisectoral, community-based rehabilitation; and universal design [17].

We are on a continual quest for global excellence by adopting key Global Initiatives and Collaboration Worldwide. Some strategies to achieve this goal are depicted in figure 3 .

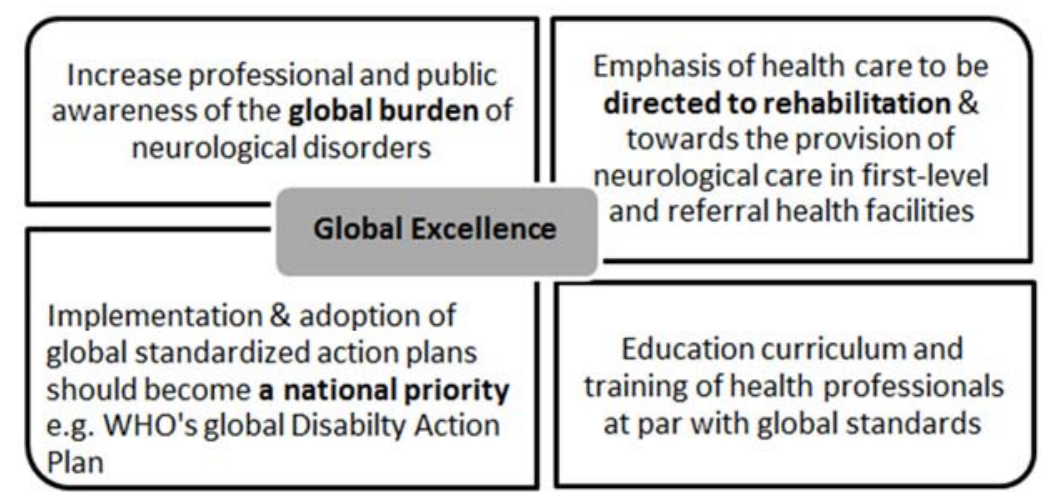

Figure 3. Global excellence.

\subsection{Policy and Planning}

Healthcare organizations and neurorehabilitation services have immense task of developing correct policies, laws, and delivery systems with coordination strategy adapted to local needs. These services may be confronted with expectations stemming from their institutional environment (e.g., government agencies, administrations, patient organizations) concerning improvement of quality of care and clinical outcomes. Policies also need to specifically promote the 
human rights of PwD [18]. Despite the burden attributed to neurological disorders, many low-middle income countries in the developing world have health policies that do not cover neurological disorders at the same level as other medical conditions [14]. Importantly, these conditions are largely absent from the national health agendas. Legislation or policies for overall management of PwD, have been adapted in many developing countries such as India, Afghanistan, Bangladesh, Sri Lanka [19], Ghana [20], Pakistan [21], Mauritius [22] and others, however, disability culture in many remains in a nascent stage [21]. The existing policies are underfunded and often there is a lack of coordination and collaboration amongst different sectors and ministries involved [23]. Despite availability of policies on rehabilitation in some countries, there is lag in implementation of these policies and/or overall delivery of the rehabilitation service [1]. Further, there is no consensus on who is responsible for enforcing and/or funding new legislations such as CRPD, the other WHO recommendations etc. [23]

On a positive note, India has made significant leaps in developing rehabilitation programs for PwD. India was one of the first countries to sign and ratify the UN Convention, and established the Rehabilitation Council of India (RCI) in 1986 [24]. In 1993, the Indian Parliament enacted the RCI Act and set it up as Statutory Body. The RCI currently regulates and monitors services given to $\mathrm{PwD}$, develops education curriculum and maintains a Central Rehabilitation Register of all qualified professionals and personnel working in the field of Rehabilitation and Special Education. The trend of year-wise status of registration of rehabilitation professionals and personnel in the Central Rehabilitation Register has quadrupled from 2001-2 (20,118 registration) to 2012-13 (78,514 registrations).

Our strategies to overcome the administrative difficulties may include (Figure 4):

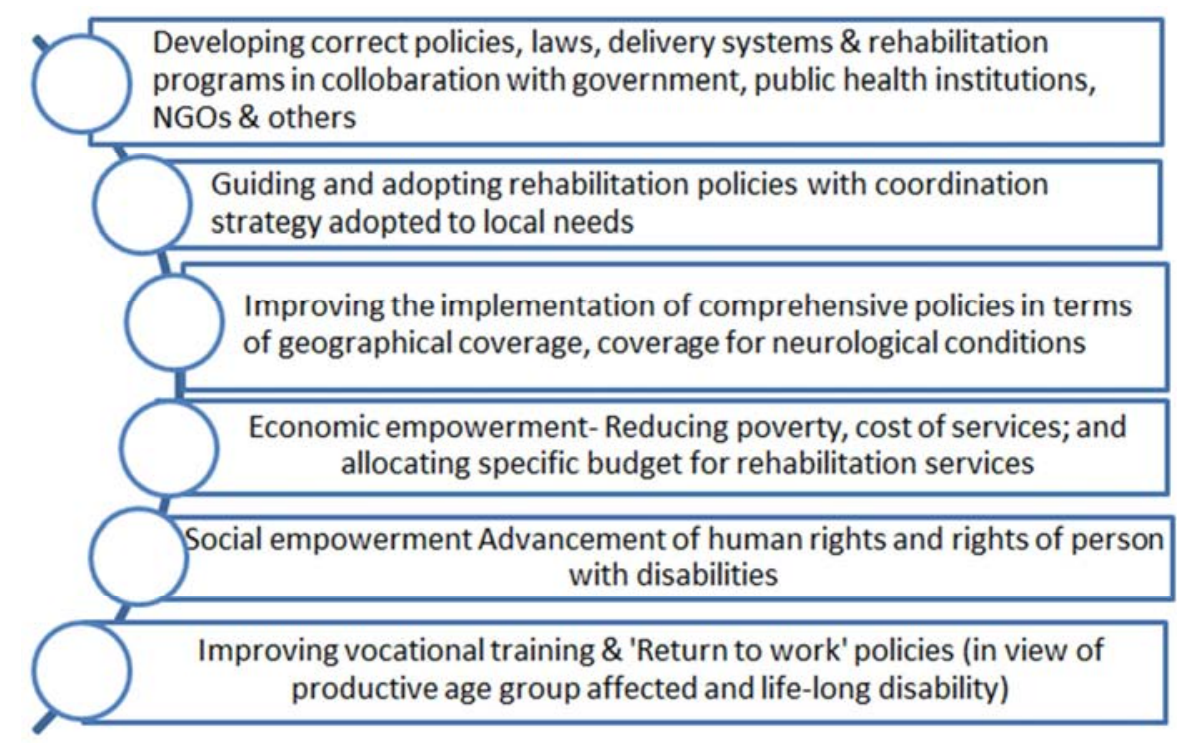

Figure 4. Policy and planning.

\subsection{Quality of Care}

Incorporating following steps may improve the quality of care provided in neurorehabilitation. (Figure 5)

Having a central body for the oversight and coordination to ensure the highest quality (such as Rehabilitation council of India)

Adoption of rehabilitation practice model rather than traditional medical model

Uniform protocols or guidelines for data collection and management of different neurological disabilities and rehabilitation

To evolve novel, innovative, needs-based, indigenously developed locally applicable, tailor made, cost effective and replicable models /programs of rehabilitation services and training for different settings 


\subsection{Resources}

With awareness of the massive burden associated with neurological disorders came the recognition that neurological services and resources were disproportionately scarce, especially in low income and developing countries. Furthermore, a large body of evidence shows that policymakers and health-care providers may be unprepared to cope with the predicted rise in the prevalence of neurological and other chronic disorders and the disability resulting from the extension of life expectancy and ageing of population globally $[7,25]$. The significant longer-term health consequences of neurological conditions in low-resource countries can overwhelm the person (caregivers), society and the health system. Lack of funding and human resources can hinder proficient implementation and management. Currently, available resources for disability in most countries are inadequate and inequitably distributed $[1,15]$. In majority of low-income countries, there are limited budgets for services for neurological conditions. The WHO survey showed only $10.4 \%$ of the responding countries (mainly high-income countries) have a separate budget for neurological illnesses within their health budgets, and two-thirds of the low income countries have no disability benefits available [15]. Out-of pocket payment by patients is the major source of financing in most low-income countries, which is likely to result in financial hardship and inequity in utilization of healthcare services. Also, when it comes to some conditions such as stroke, the cost spent for brain scan and other such procedures for acute management is huge. So when they come for rehabilitation, they are left with nothing other than low cost rehabilitation.

Further, the number of trained professionals handling the millions of people with neurological disabilities is miniscule. It is estimated that in India, there are about 2000 neurologists, 600 physiatrists, a few thousand therapists and a dozen neuropsychologists catering to a population of over a billion people, with services available primarily in major cities [26].

Expanding Resources- Our funding and human resources should be adequate and equitably distributed. (Figure 6)

\begin{tabular}{|c|c|}
\hline Funding resources & Human resources \\
\hline $\begin{array}{l}\text { New legislatives- such as } \\
\text { Universal Health Coverage }\end{array}$ & $\begin{array}{l}\text { Developing leadership, } \\
\text { workforce \& capacity by training } \\
\text { specialists professionals }\end{array}$ \\
\hline $\begin{array}{l}\text { Seperate budget for } \\
\text { Neurological illnesses \& } \\
\text { rehabilitation services within } \\
\text { the health budget }\end{array}$ & $\begin{array}{l}\text { Appropriate distribution } \\
\text { between urban \& rural regions }\end{array}$ \\
\hline Improving funding mechanisms & $\begin{array}{l}\text { New partnerships and strategic } \\
\text { collaboration }\end{array}$ \\
\hline $\begin{array}{l}\text { Insurance policies; mobilising } \\
\text { funds from grants, aids; thus } \\
\text { reducing the out-of-pocket } \\
\text { source (as the current source of } \\
\text { funding) }\end{array}$ & $\begin{array}{l}\text { Involvement of bilateral } \\
\text { agencies using multi-sectoral } \\
\text { approach }\end{array}$ \\
\hline Disability benefits available & \\
\hline
\end{tabular}

Figure 6. Expanding resources.

\subsection{Enabling Infrastructure and Physical Resources}

Many persons with neurological conditions require highly specialized skills for appropriate diagnosis and management. Most developing nations lack or have limited number of adequate and efficient health services, particularly in rural areas [27]. In these countries sub-specialized services (such as stroke units) are restricted to urban areas [15]. The availability of rehabilitation services across regions varies, and many developing countries still have limited or no organized rehabilitation services [1]. In many, these services exist through NGO initiatives and charitable organizations; these are often dependent on external funding, independent of national public health systems [19]. There is minimal data collection, reporting and information gathering system, which impedes evaluation and monitoring of the situation over-time to alert the health system to emerging trends, and to facilitate planning [13].

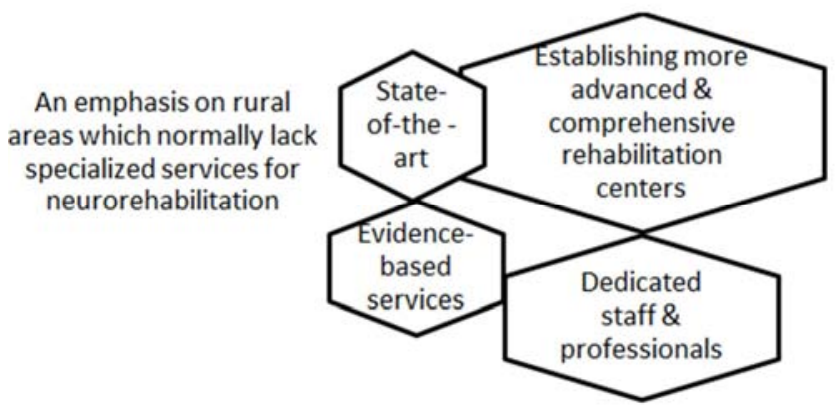

Figure 7. Enabling Infrastructure.

\subsection{Improving Emergency Care (Figure 8)}

Stroke is one of the leading causes of death and disability in India. There are no comprehensive stroke units and limited centers in the country offer thrombolysis and coordinated stroke services. Majority of stroke cases are managed in general medical wards, which often lack follow up, plan or referral for stroke rehabilitation [26]. Also, India has become the Head Injury Capital of the World due to road traffic accidents.

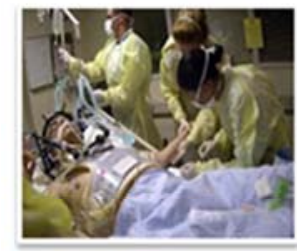

Early and prompt intervention will help in reducing the impact neurological damage (in conditions like stroke, traumatic brain or spinal cord injury, etc.) and will subsequently improve the long-term outcome of Neurorehabilitation.

Figure 8. Emergency care.

\subsection{Health Information Systems}

In the developing world, healthcare systems are limited by resources, capability and lack of information technology 
systems. Data on neurorehabilitation is incomplete and fragmented, and often not disaggregated from other healthcare services information [28]. There is lack of routinely collected data for disability, limited information about the needs and unmet needs of PwD, variability in definitions used for disability and various types of disability (e.g., physical, mental, behavioral, intellectual) and variation in settings [1, 17]. Further, there is diversity in models of care, interventions and modalities, outcome measurement, type and quality within neurorehabilitation services. Healthcare systems and practices vary amongst countries. Despite availability of the ICF for over a decade, there is no indicator or common framework for describing and measuring rehabilitation in many countries; and rehabilitation measures are often excluded from existing classification systems [1]. Inadequate health information systems and communication strategies across health care sectors and between providers (notably between primary and secondary care), is still prominent worldwide, even in developed countries like Australia [29]. Other barriers to provision of neurorehabilitation services, include, inconsistent and insufficient data collection processes, multiple clinical information systems and incompatible technologies [1,29].
Our aim is to have complete, consistent \& uniform data by implementing following steps. (Figure 9)
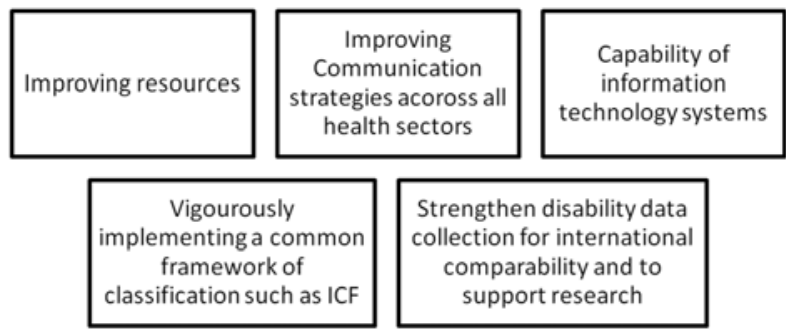

Strengthen disability data collection for international comparability and to support research

Figure 9. Strategies for an Efficient Health Information System.

\subsection{Referral Systems}

Many developing countries have no systems of appropriate referral mechanism for neurological illnesses. Therefore, many people miss appropriate services and/or care continuum in the community, after discharged from an acute facility. An effective referral system (figure 10) is required to ensure a close relationship/coordination between all levels of the health system to ensure those with neurological disorders, receive the best possible care and regular follow-up [14, 30].

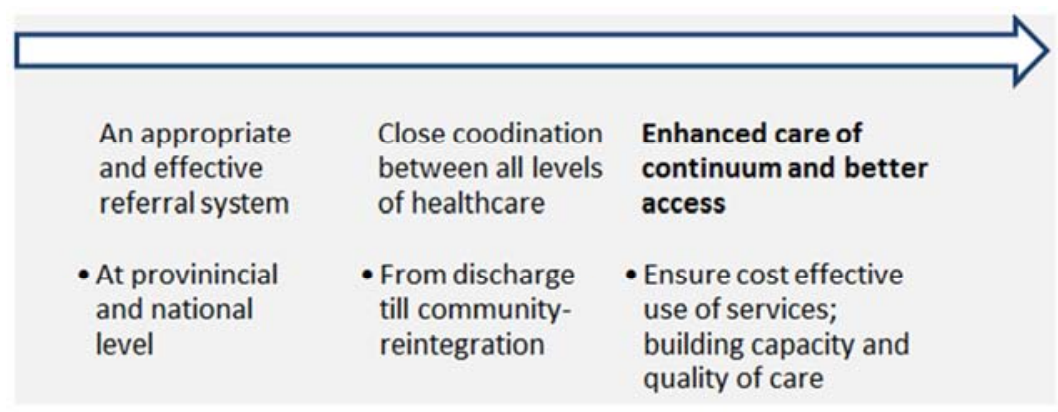

Figure 10. Effective Referral system.

\section{Community Support}

\subsection{Need for Professional, Caregiver and Consumer Groups: (Figure 11)}

Though there is an increasing trend in the number of professional, caregiver groups and NGOs, for PwD worldwide, there is limited number of such organizations for disabled population in low-income countries. According to WHO, there are no NGOs or other professional groups for neurological disorders in $35 \%$ of low-income countries [15].

\begin{tabular}{|l|l|l|}
$\begin{array}{l}\text { WHOs "Bottom- } \\
\text { up" approach, } \\
\text { through } \\
\text { community- } \\
\text { based \& multi- } \\
\begin{array}{l}\text { sectoral } \\
\text { rehabilitation }\end{array}\end{array}$ & $\begin{array}{l}\text { Increasing the number of } \\
\text { professional, caregiver } \\
\text { groups, support groups, } \\
\text { community groups and } \\
\text { NGOs and such } \\
\text { organizations for persons } \\
\text { with neurorehabilitation } \\
\text { worldwide. }\end{array}$
\end{tabular}

Figure 11. Professional, caregiver and consumer groups

\subsection{Multidisciplinary Team for comprehensive care}

An additional challenge for neurorehabilitation is that people with neurological conditions have concurrent physical, speech, cognitive and behavioral issues requiring specialized services for comprehensive management. Neurorehabilitation team represents multidisciplinary body including neurologists, neurosurgeons, physiatrists, 
neuropsychologists, physical therapists, occupational therapists, speech therapists, and other professionals taking care of people with neurological disabilities. A casual observation would reveal that in the few health facilities that parade a reasonably constituted neurorehabilitation team, intraprofessional communication and relationship is far from adequate [31]. The idea of a cyclical relationship among all team members with the patient as the focus is alien. The ideal team work approach may involve the necessary considerations as depicted in figure 12 .

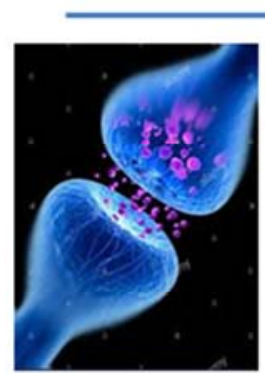

Strengthening our synapses to work together as an integrated, multidisciplinary team for a coordinated care

Sharing and adopting the standards of behavior that bring out the best in us both individually and collectively

Having an honest and open communication and information system and environment of respect and trust where all health professionals thrive as a team

Even other sectors should be a part of the team e.g. engineers, architects for building barrier-free physical environment

Figure 12. Teamwork.

\subsection{Improved Awareness and Education}

(Figure 13) The concept of rehabilitation is confused with 'Physiotherapy and exercises' alone by many health care professionals and general public. Majority of people with disabilities, are poorly educated and unaware of specialties like rehabilitation; and information about benefits of rehabilitation are not easily available or understandable [1, $31,32]$. Lack of mistrust among patients for medical practitioners in some cultures is still prevalent, particularly in rural areas, where first preference is to seek help from religious healers and traditional herbal medicine.

It is necessary to propagate the important concept of impairment management and prevention of disability in patients with neurological diseases. Holding awareness programs for survivors with disabilities occurring as a result of neurological disorders can be fruitful. Our aim is to set up an effective communication strategy to encourage dialogue and we work to make sure we hear from and talk to these audiences in the right way and at the right time, as an important part of the care we provide for our community.

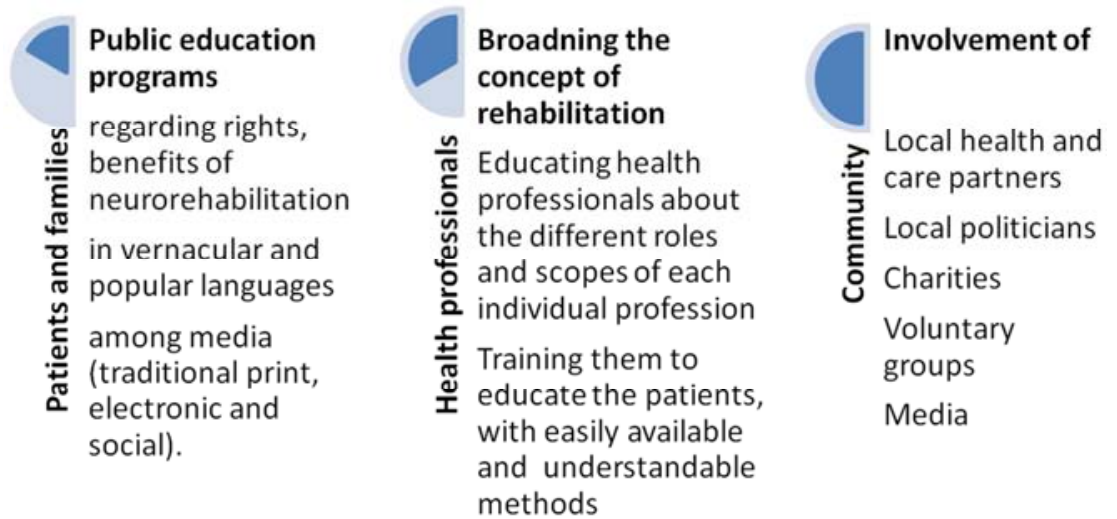

Figure 13. Strategies to improve awareness and education.

\subsection{ICF and Community Re-integration}

Even after regaining most of the sensori-motor functions, people with neurological conditions do not regain the ability to participate in the society. In a study on spinal cord injury in rural South India reduced level of community reintegration was attributed to architectural and environmental barriers, poor socioeconomic status and comorbidities [33]. Our aim is to ensure that each patient achieves the maximum functional recovery and enable them to lead independent, productive, dignified and meaningful lives in the community. Endorsing ICF model as an outcome will help to improve involvement in their role in life and participation in the community. 


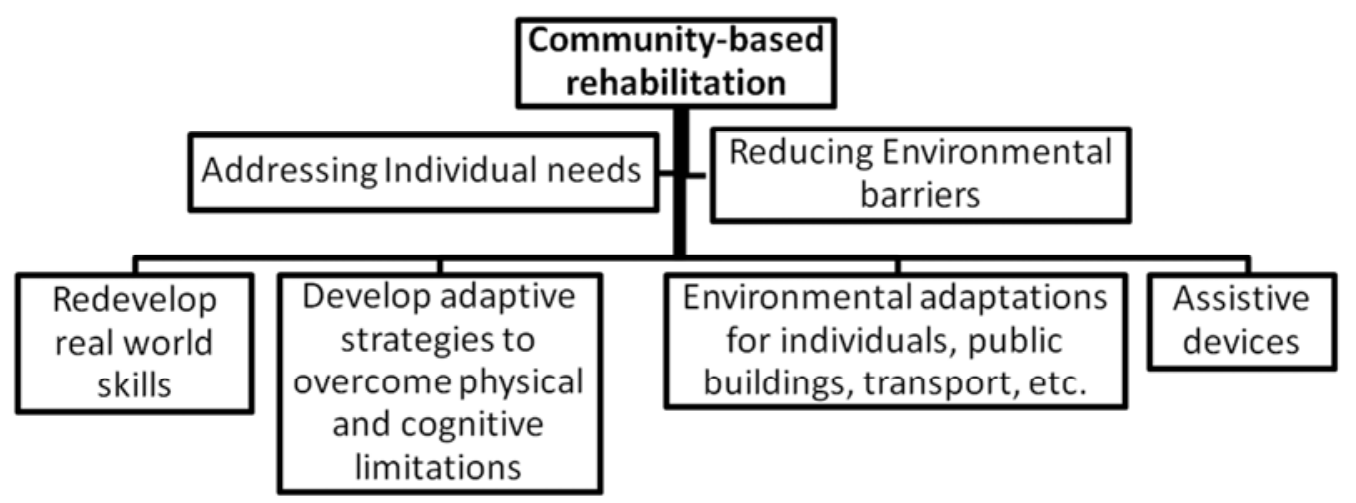

Figure 14. Community re-integration.

\subsection{Client and Family-centered Approach}

India has a long tradition of implementing CBR programs, involving the families and caregivers with good effect using local traditions. Due to unique socio-demographic profile of our country, it is essential to preserve the social fabric and cultural values in this diverse population by valuing the life, ideas, and beliefs of every individual, treating each person with dignity and respect; catering to their individual as well as culture-specific needs. A platform is required to create advocacy for the people and families suffering from neurological disease with disability and involving them in decision making.

\subsection{Integrated and Holistic Care}

Neurological conditions can cause complex disabilities, are costly to treat and have socioeconomic implications due to increased demand for health care, social and vocational services, and caregiver burden [32]. Longer-term functional and psychosocial issues limit daily activity and participation. These require integrated interdisciplinary collaborative care in hospital and community to enable these people to live independently in the community. Thus, it is important to integrate our approach in a holistic manner with the different aspects of Neurorehabilitation needs, viz.

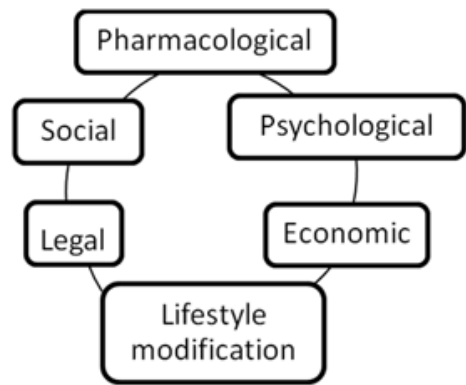

Figure 15.Collaborative care

\subsection{Psychological Barriers}

Despite evidence of growing public acceptance of PwD, widespread stigma and discrimination still persists, often because of non-biologic concepts and cultural perception of disabilities [1] (Figure 16). This has resulted in unsuccessful implementation of the World Report on Disability's explicit recommendations for social inclusion such as communitybased rehabilitation in African countries [34, 35].

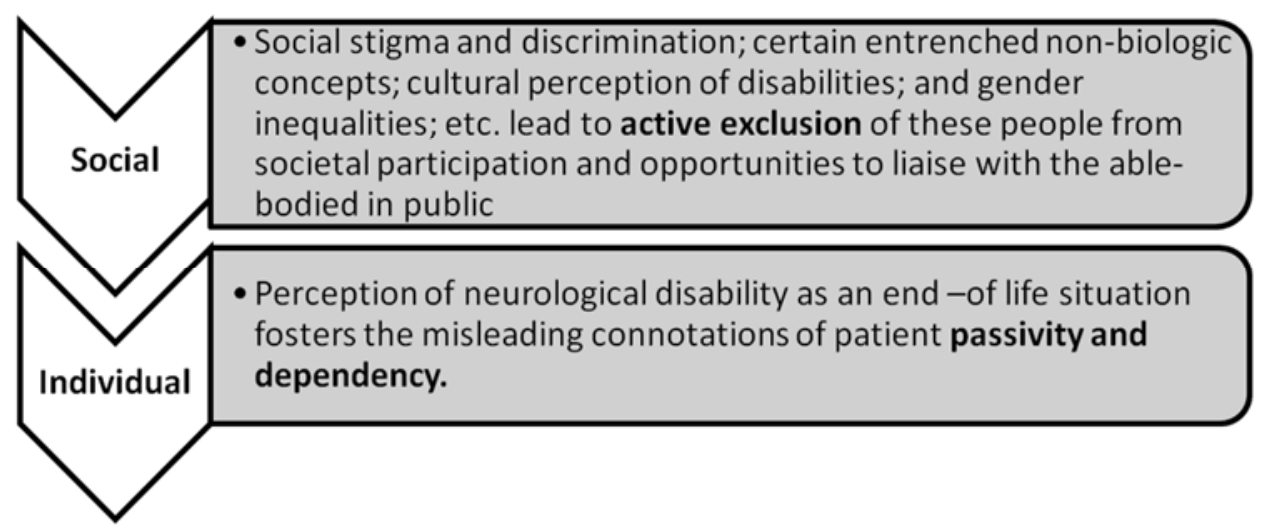

Figure 16. Psychological barriers prohibit social inclusion.

Stigma and discrimination against people with neurological disorders are ubiquitous and need to be eliminated through public education and global campaigns. (Figure 17) 


\section{Addressing the Psychological barriers for successful integration}

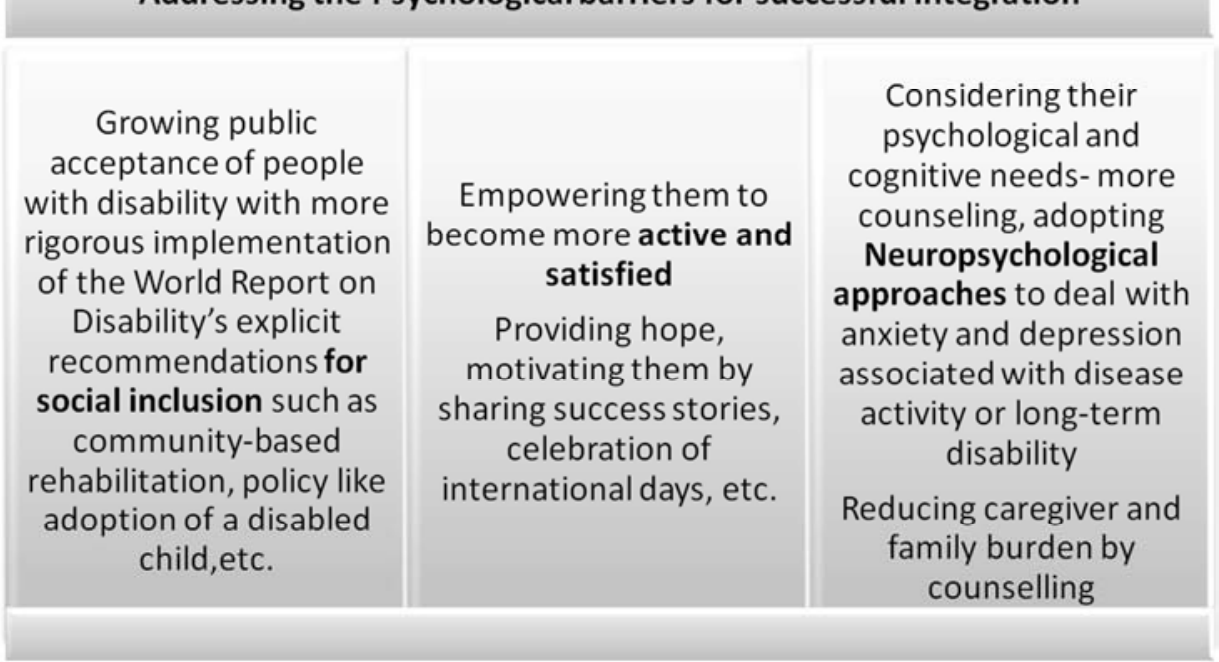

Figure 17. Strategies for Psychosocial inclusion

\subsection{Research}

Traditional Neurorehabilitation procedures appear to have limited efficacy in common neurological diseases. Recent lines of research on neuroplasticity and recovery have furthered our knowledge and have stimulated novel rehabilitation paradigms. Further investigation to evaluate the new developments and refine the extant literature is needed to pursue the best outcomes and excellence in healthcare delivery. Corollary, it is important to promote clinical research in the field of neurorehabilitation. Also, research should move beyond the simple question of whether rehabilitation is effective and feasible, and examine the therapy factors and patient characteristics that optimize the clinical outcomes of rehabilitation. It is important to organize conferences/workshops/symposiums for neurorehabilitation professionals to train/share the knowledge/scientific research/advanced procedures training to keep abreast with the recent advances and work done in the field. More research on neurological disorders is needed and it should be facilitated through better funding, multidisciplinary approaches and international collaboration. (Figure 18)

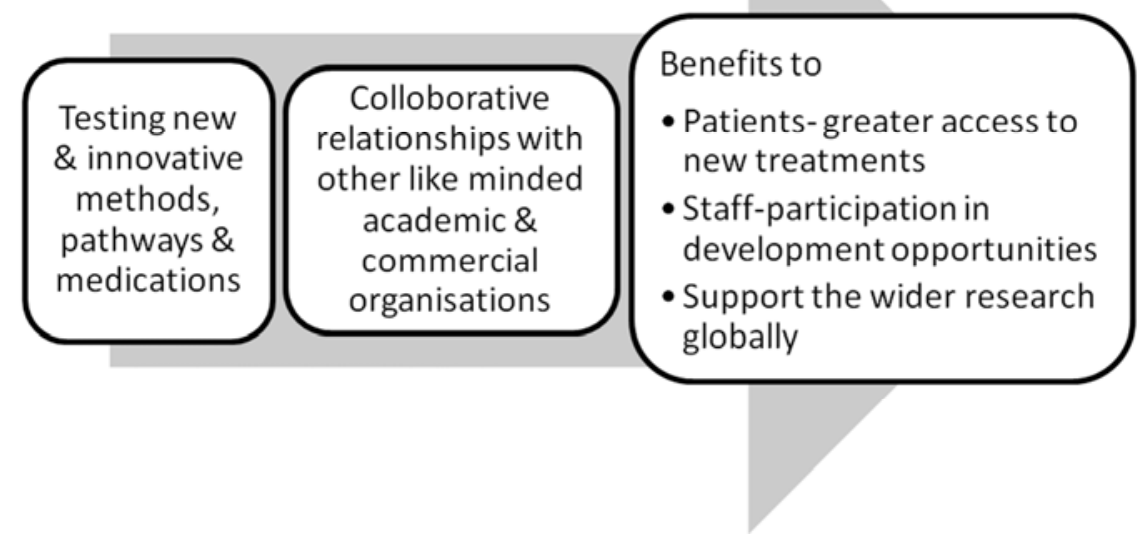

Figure 18. Directions for Research.

\subsection{Technology in Neurorehabilitation}

With evolution in science and technology, we have a new toolbox of options to provide cutting edge improvements to therapies accelerating the pace of Neurorehabilitation. Converging clinical and engineering research should leverage scientific discoveries and further enhance the current clinical practice, develop effective and patient-tailored rehabilitative approaches and maximize the functional outcome. (Figure 19) 


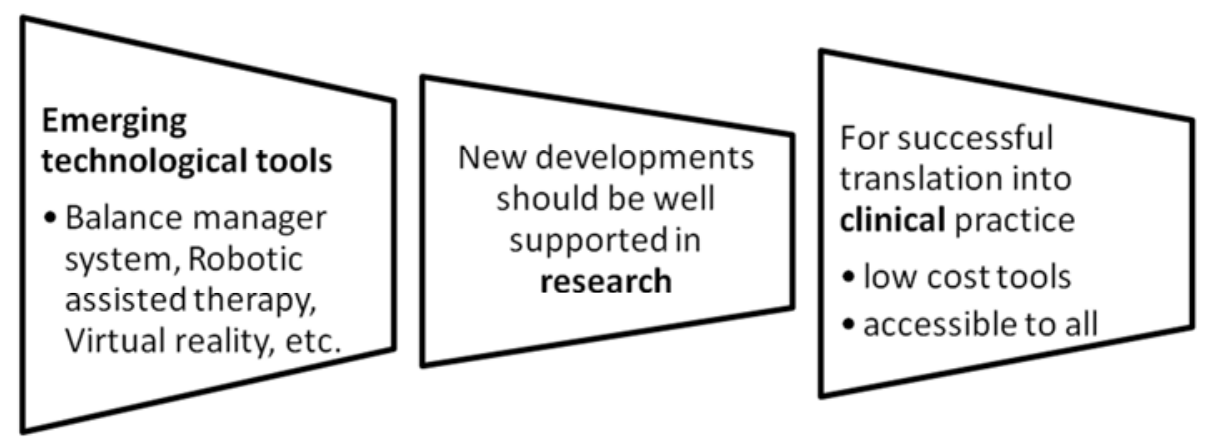

Figure 19. Technology in Neurorehabilitation.

\subsection{Academics and the Future Professionals}

Developing countries don't have educational programs for rehabilitation professionals. Also, education-commonly institutional and urban-based is not always relevant to rural reality. Our vision is to impart training to create empowered manpower to manage persons with neurological disability. Neurorehabilitation being a specialized field in itself, extra efforts with investment in education and skill development for the next generation of health care providers is essential.
It's important to acknowledge that students and health professionals perceive dealing with neurological conditions 'blood-brain barrier' (complex and challenging)! Initiatives will be taken to develop their inclination towards this field by creating more opportunities to share the emerging knowledge with practicing professionals and experts through many educational activities.

Following initiatives are essential in the successful implementation. (Figure 20)

\section{Education institutes Need of the hour}

- Having specialized rehabilitation departments in medical schools and hospitals

- Formal training of undergraduate medical students and allied health faculties

\section{Education Curriculum and Training $\begin{aligned} & \text { Bridging the gap of knowledge and clinical } \\ & \text { exposure }\end{aligned}$}

- Review present curricula keeping in mind the emergence of new technologies for imparting education and rehabilitation services.

- Conducting more and specialized workshops

\section{Inclination of Students and Health professionals}

\section{Rewarding}

- Providing incentives in the form of scholarships

- Making the training more rewarding in terms of job opportunities

- Preparing them for the psycholgically challenging prerequisites viz. consistency, patience, determination, etc

Figure 20. Initiatives for academic and professional growth

\section{Conclusion}

All the above mentioned disparate threads need to be woven together to reflect the integrated nature of Neurorehabilitation!

Bringing in a hope of ameliorating the burdens placed on the patients as well as their family by diseases that change the very way we live is simply incredible; we can give people their lives back. If your goal as a health professional is to bear witness to humanity at its best and worst, Neurorehabilitation will certainly give you that opportunity! Articulating our aspirations through this 'Vision
Neurorehabilitation $2025^{\prime}$ we lay down the framework to bring out a systematic change through a holistic and integrated approach with determination and perseverance to provide even better future for our patients. On the platform of International Journal of Neurologic Physical Therapy, I wish success to the ever emerging field of Neurosciences and Neurorehabilitation! I am sure this field will experience growth like no other and will become 'the final frontier of Medicine and Rehabilitation'!! Let us strive and join together to bring enrichment to our vision, achievements and a continuous progress of Neurorehabilitation. 


\section{Abbreviations}

CBR: Community based rehabilitation

CRPD: Convention on the Rights of Persons with Disabilities

ICF: International Classification of Functioning, Disability and Health

NGO: Non-Governmental Organization

PwD: Persons with disability

RCI: Rehabilitation Council of India

WFN: World Federation of Neurology

WHO: World Health Organization

\section{References}

[1] World Health Organization. World Report on Disability. Geneva: WHO; 2011.

[2] Department of Health. National Services Framework (NSF) for Long-term Conditions. Leeds: DH; March 2005.

[3] Australian Rehabilitation Alliance. The need for a National Rehabilitation Strategy: working towards a clear and united rehabilitation strategy for Australia (Position Statement). Canberra: ARA; 10 August 2011.

[4] Khan F, Amatya b, NgL, Drummond K, Olver J. Multidisciplinary rehabilitation after primary brain tumour treatment. Cochrane Database Syst Rev. 2013; 1: CD009509.

[5] United Nations General Assembly. Keeping the promise: realizing the Millennium Development Goals for persons with disabilities towards 2015 and beyond: Report of the SecretaryGeneral, New York: UN; 26 July 2010.

[6] World Health Organization. The International Classification of Functioning, Disability and Health. Geneva: WHO; 2001.

[7] World Health Organization. Promoting access to healthcare services for persons with disabilities, in Disability and rehabilitation. Geneva: WHO; 2006.

[8] South-North Centre for Dialogue and Development. Global survey of government actions on the implementation of the standard rules of the equalisation of opportunities for persons with disabilities. Amman: Office of the UN Special Rapporteur on Disabilities; 2006.

[9] Elrod CS, DeJong G. Determinants of utilization of physical rehabilitation services for persons with chronic and disabling conditions: an exploratory study. Arch Phys Med Rehabil. 2008; 89: 114-120.

[10] Eide AH, Øderud T. Assistive technology in low income countries. In: Maclachlan M, Swartz L, editors. Disability and international development. Dordrecht, Netherlands: Springer; 2009.

[11] Mitra S, Findley PA, Sambamoorthi U. Health care expenditures of living with a disability: total expenditures, out-of-pocket expenses, and burden, 1996 to 2004. Arch Phys Med Rehabil. 2009; 90: 1532-1540.

[12] Mitra S, Sambamoorthi U. Disability prevalence among adults: estimates for 54 countries and progress toward a global estimate. Disabil Rehabil. 2014; 36: 940-947.
[13] Parnes P, Cameron D, Christie N, Cockburn L, Hashemi G, Yoshida K. Disability in low-income countries: issues and implications. Disabil Rehabil. 2009; 31: 1170-1180.

[14] World Health Organization. Neurological disorders: public health challenges. Geneva: WHO; 2006.

[15] World Health Organization and World Federation of Neurology. Atlas: country resources for neurological disorders. Geneva: WHO; 2004.

[16] Janca A, Prilipko L, Costa e Silva JA. The World Health Organization's work on public health aspects of neurology. J Neurol Neurosurg Psychiatry. 1997; 63 Suppl 1: S6-7.

[17] World Health Organization. WHO global disability action plan 2014-2021: Better health for all people with disability. In: Sixty-seventh world health assembly- A67/16. Provisional agenda item 13.3. Geneva: WHO; 2014.

[18] Mannan H, Eltayeb S, Maclachlan M, Amin M, McVeigh J, Munthali A, et al. Core concepts of human rights and inclusion of vulnerable groups in the mental health policies of Malawi, Namibia, and Sudan. Int J Ment Health Syst. 2013; 7: 7.

[19] Axelsson C. Mapping report of physical rehabilitation services in Afghanistan, Bangladesh, Odisha (India) \& Sri Lanka., Handicap International, 2014.

[20] Tuakli-Wosornu YA, Haig AJ. Implementing the World Report on Disability in West Africa: challenges and opportunities for Ghana. Am J Phys Med Rehabil. 2014; 93: S50-57.

[21] Rathore FA, New PW, Iftikhar A. A report on disability and rehabilitation medicine in Pakistan: past, present, and future directions. Arch Phys Med Rehabil. 2011; 92: 161-166.

[22] Soopramanien A. Mauritius calling: medical care and neurorehabilitation needs in an oceanic idyll. Arch Phys Med Rehabil. 2012; 93: 2377-2381.

[23] World Health Organization. Disability and rehabilitation status review of disability issues and rehabilitation services in 29 African Countries. Geneva: WHO; 2004.

[24] Rehabilitation Council of India. 26th Annual Report 2012-13. New Delhi: RCI; 2013.

[25] Bethge M, von Groote P, Giustini A, Gutenbrunner C. The World Report on Disability: a challenge for rehabilitation medicine. Am J Phys Med Rehabil. 2014; 93: S4-11.

[26] 10th World Congress For Neurorehabilitation From Neurotechnologies To Community Care. http://www.wenr2018.in/about_ifnr.html.

[27] World Health Organization, Swedish Organizations of Disabled Persons International Aid Association. Part 1. Community-Based Rehabilitation as we experienced it voices of persons with disabilities. Geneva: WHO; 2002.

[28] Worrall LE, Howe T, O'Callaghan A, Hill AJ, Rose M, Wallace SJ, et al. The World Report on Disability as a blueprint for international, national, and local aphasia services. Int J Speech Lang Pathol. 2013; 15: 106-112.

[29] Digiacomo M, Davidson PM, Taylor KP, Smith JS, Dimer L, Ali $M$, et al. Health information system linkage and coordination are critical for increasing access to secondary prevention in Aboriginal health: a qualitative study. Qual Prim Care. 2010; 18: 17-26. 
[30] Turner-Stokes L, Sykes N, Silber E; Guideline Development Group. Longterm neurological conditions: management at the interface between neurology, rehabilitation and palliative care. Clin Med. 2008; 8: 186-191.

[31] Hamzat TK. Some Challenges Facing Neurorehabilitation in Nigeria: Standpoint of a Neurophysiotherapist. Journal of Neurology and Neurorehabilitation Research. 2016; 1 (1).

[32] McAllister L, Wylie K, Davidson B, Marshall J. The World Report on Disability: an impetus to reconceptualize services for people with communication disability. Int J Speech Lang Pathol. 2013; 15: 118-126).

[33] Sekaran P, Vijayakumari F, Hariharan R, Zachariah K, Joseph
SE, Senthil Kumar RK. Community Reintegration of Spinal Cord-injured Patients in Rural South India. Spinal Cord. 48 (8): 628-32.

[34] Kuyini AB, Alhassan AR, Mahama FK. The Ghana community-based rehabilitation program for people with disabilities: what happened at the end of donor support? J Soc Work Disabil Rehabil. 2011; 10: 247-267.

[35] Turner-Stokes L, Sykes N, Silber E, et al. From diagnosis to death: exploring the interface between neurology, rehabilitation and palliative care in managing people with long-term neurological conditions. Clin Med. 2007; 7: 129136. 